The similarity of lithium transference numbers at low salt concentrations in aqueous solutions and the gelled electrolytes, the case for which direct comparison should be valid, lends credence to our claim that the ENMR method yields essentially correct measurement of transference in these systems. Furthermore, the trends in transference number with concentration and counterion size are in accord with reasonable expectations.

\section{Conclusion}

We have described the use of a new method for the determination of lithium transference numbers in polymer gel systems. Conceptually, the method probes the underlying ion motions which lead to concentration polarization in the Hittorf method. Important advantages of the method described are its rapidity, relative simplicity, and relative freedom from complicating factors related to electrode processes. By straightforward extension, this method should be useful for the accurate determination of net transference numbers in polymer electrolytes, a task which has proved difficult. More detailed studies of lithium ionconducting polymer electrolytes are currently underway in our laboratory.

\section{Acknowledgment}

This work was supported by the U.S. Department of Energy Office of Transportation Technology. We also thank Professor Eric McFarland of University of California/Santa Barbara for helpful discussions.

Manuscript received March 4, 1996.

Los Alamos National Laboratory assisted in meeting the publication costs of this article.

\section{REFERENCES}

1. F. Gray, Solid Polymer Electrolytes Fundamentals and Technological Applications, VCH, New York (1991).

2. P. G. Bruce and C. A. Vincent, Faraday Discuss. Chem. Soc., 88 , 43 (1989).

3. H. P. Fritz and A. Kuhn, J. Power Sources, 41, 253 (1993).

4. P. G. Bruce, M. T. Hardgrave, and C. A. Vincent, Solid State lonics, 53, 1087 (1992).

5. M. Holz, Chem. Soc. Rev., 165 (1994).

6. C. S. Johnson, Jr. and Q. He, Adv. Magn. Reson., 13, 131 (1989).

7. R. A. Robinson and R. H.Stokes, Electrolyte Solutions, Butterworths, London (1959).

\title{
Effect of Interfacial Resistance on Determination of Transport Properties of Mixed-Conducting Electrolytes
}

\author{
Meilin Liu* and Hongxing $\mathrm{Hu}$ \\ School of Materials Science and Engineering, Georgia Institute of Technology, Atlanta, Georgia 30332-0245, USA
}

\section{ABSTRACT}

Combination of impedance spectroscopy and open-cell voltage measurements has been used to determine the transport properties of mixed-conducting electrolytes. It is clearly demonstrated experimentally that the ratio of open-cell voltage to the applied Nernst potential can be quite different from the average ionic transference number; the effect of interfacial polarization resistance on determination of transference numbers of a mixed-conducting electrolyte must be carefully analyzed, particularly for a cell based on a thin electrolyte, tested at low temperatures, or exposed to an atmosphere containing low activities of electroactive species.

\section{Introduction}

While the total conductivity of a mixed ionic-electronic conductor (MIEC) is readily obtainable, separation of the ionic from the electronic conductivity is often complicated by the interfacial polarizations associated with mixed conduction. Yet, the effect of interfacial polarization on the determination of transport properties of an MIEC has traditionally been ignored in the past. For an MIEC with significant ionic conduction (i.e., a mixed-conducting electrolyte), for instance, the average ionic transference number is usually calculated from the following equation ${ }^{1-3}$

$$
t_{i}=\frac{V_{\alpha}}{E_{N}}
$$

where $V_{o c}$ is the open-cell voltage (OCV) across the MIEC while a Nernst potential $\left(E_{\mathrm{N}}\right)$ is imposed across the electrolyte. The $E_{\mathrm{N}}$ is determined by the activities of electroactive species at the electrode-electrolyte interfaces. For a cell based on a $\mathrm{BaCe}_{0.8} \mathrm{Gd}_{0.2} \mathrm{O}_{3}$ [gadolinia-doped barium cerate (BCG)] electrolyte with a configuration of $p_{\mathrm{O}_{2}}^{\prime}$, $\mathrm{Pt}$ | $\mathrm{BCG} \mid \mathrm{Pt}, p_{\mathrm{O}_{2}}^{\prime \prime}$, the $E_{\mathrm{N}}$ is given by

$$
E_{\mathrm{N}}=\frac{R T}{4 F} \ln \left(\frac{p_{\mathrm{O}_{2}}^{\prime \prime}}{p_{\mathrm{O}_{2}}^{\prime}}\right)
$$

where $p_{\mathrm{O}_{2}}^{\prime}$ and $p_{\mathrm{O}_{2}}^{\prime}$ are the partial pressures of oxygen at the two interfaces of the cell.

Equation 1 was derived, for the first time, by Wagner ${ }^{4}$ under the assumption that the interfacial processes are infinitely fast. However, this equation has been used frequently in the literature to calculate the average ionic transference numbers of an MIEC under various conditions. The assumption of infinitely fast interfacial processes may not be valid in general and in many cases it is the interfacial resistance which dictates the performance of a solidstate ionic device, particularly for a cell based on a thin-film electrolyte or operated at low temperatures. ${ }^{5}$

Earlier analysis indicated that the OCV across an MIEC is influenced by the overpotentials developed at the interfaces $\left(\eta_{a}-\eta_{c}\right)$ or by the interfacial resistance $\left(R_{\mathrm{p}}\right)$ of the cell as follows ${ }^{6}$

$t_{\mathrm{i}}=\frac{V_{\mathrm{oc}}}{E_{\mathrm{N}}}\left[1-\frac{\eta_{\mathrm{a}}-\eta_{\mathrm{c}}}{E_{\mathrm{N}}}\right]^{-1}=\frac{V_{\mathrm{oc}}}{E_{\mathrm{N}}}(1+\delta)$ where $\delta=\frac{R_{\mathrm{p}}}{R_{\mathrm{i}}+R_{\theta}}$

where $R_{\mathrm{i}}$ and $R_{\mathrm{e}}$ are the average ionic and electronic resistances of the bulk electrolyte, respectively. Theoretically, $t_{i}$ can be calculated accurately using Eq. 3. In practice, however, neither $\eta_{a}-\eta_{c}$ nor $R_{\mathrm{p}}$ is easily obtainable from a single experiment.

Recently, it is demonstrated that the combination of impedance spectroscopy and OCV (or gas permeation) measurements allows accurate determination of $R_{\mathrm{i}}, R_{\mathrm{e}}$, and $R_{\mathrm{p}}{ }^{7}$ From an impedance spectrum, the total resistance of a cell $\left(R_{T}\right)$ and the resistance of the bulk electrolyte $\left(R_{b}\right)$ can be determined readily with good confidence. In a Nyquist plot, ${ }^{8}$ for example, the intercept of the impedance loop with the real-axis at high frequencies represents $A_{b}$

$$
\lim _{w \rightarrow \infty}\{Z\}=R_{\mathrm{b}}=\frac{R_{\mathrm{i}} R_{\mathrm{e}}}{R_{\mathrm{i}}+R_{\theta}}
$$

while the intercept of the impedance loop with the real-axis at low frequencies corresponds to $R_{\mathrm{T}}$

$$
\lim _{\omega \rightarrow 0}\{Z\}=R_{\mathrm{T}}=\frac{\left(R_{\mathrm{i}}+R_{\mathrm{p}}\right) R_{\mathrm{e}}}{\left(R_{\mathrm{i}}+R_{\mathrm{p}}\right)+R_{\mathrm{e}}}
$$

Solving for $R_{\mathrm{i}}, R_{\mathrm{e}}$, and $R_{\mathrm{p}}$ from Eq. 3, 4, and 5, we have

$$
R_{\theta}=\frac{R_{\mathrm{T}}}{1-\frac{V_{\mathrm{oc}}}{E_{\mathrm{N}}}}
$$




$$
\begin{gathered}
R_{\mathrm{i}}=\frac{R_{\mathrm{b}} R_{\mathrm{e}}}{R_{\mathrm{e}}-R_{\mathrm{b}}}=\frac{R_{\mathrm{b}}}{1-\frac{R_{\mathrm{b}}}{R_{\mathrm{T}}}\left(1-\frac{V_{\mathrm{oc}}}{E_{\mathrm{N}}}\right)} \\
R_{\mathrm{p}}=\frac{R_{\mathrm{T}} E_{\mathrm{N}}}{V_{\mathrm{oc}}}-R_{\mathrm{i}}=\frac{R_{\mathrm{T}}-R_{\mathrm{b}}}{\frac{V_{\mathrm{oc}}}{E_{\mathrm{N}}}\left[1-\frac{R_{\mathrm{b}}}{R_{\mathrm{T}}}\left(1-\frac{V_{\mathrm{oc}}}{E_{\mathrm{N}}}\right)\right]}
\end{gathered}
$$

where $V_{\mathrm{oc}}$ and $E_{\mathrm{N}}$ from an OCV measurement as well as $\boldsymbol{R}_{\mathrm{b}}$ and $\boldsymbol{R}_{\mathrm{T}}$ from an impedance measurement must be determined from the same cell under identical conditions. The average ionic transference number of the electrolyte, then, can be calculated as

$$
t_{\mathrm{i}} \equiv \frac{R_{\mathrm{e}}}{R_{\mathrm{i}}+R_{\mathrm{e}}}=1-\frac{R_{\mathrm{b}}}{R_{\mathrm{T}}}\left(1-\frac{V_{\mathrm{oc}}}{E_{\mathrm{N}}}\right)
$$

The approximation of this equation by Eq. 1 introduces a relative error of

$$
\epsilon=\frac{\eta_{\mathrm{a}}-\eta_{\mathrm{c}}}{E_{\mathrm{N}}}=\frac{\delta}{1+\delta}=1-\frac{\frac{V_{o c}}{E_{\mathrm{N}}}}{1-\frac{R_{\mathrm{b}}}{R_{\mathrm{T}}}\left(1-\frac{V_{o c}}{E_{\mathrm{N}}}\right)}
$$

Here, BCG electrolyte is taken as an example to illustrate how this relative error $\epsilon$ (and hence the validity of Eq. 1) is influenced by experimental conditions such as temperature, $p_{0_{2}}$, and cell geometry. It is demonstrated experimentally that $\mathrm{Eq} .1$ is invalid for cells based on a BCG electrolyte under all conditions tested in this study.

\section{Experimental}

$\mathrm{BaCe}_{0.8} \mathrm{Gd}_{0.2} \mathrm{O}_{3}$ (BCG) electrolyte disks were prepared as described elsewhere ${ }^{\theta}$ to achieve sintered density greater than $95 \%$ of the theoretical value. Platinum paste (Heraeus-OS2) was screen-printed on both sides of each electrolyte disk (thickness $1.2 \mathrm{~mm}$ and diam $15 \mathrm{~mm}$ ) and subsequently fired in air at $1200^{\circ} \mathrm{C}$ for $30 \mathrm{~min}$ to form porous $\mathrm{Pt}$ electrodes (the electrode area was $0.91 \mathrm{~cm}^{2}$ ). All impedance and potentiometric measurements ${ }^{8}$ were performed on a concentration cell of

$$
\text { air, } \mathrm{Pt}|\mathrm{BCG}| \mathrm{Pt}, p_{\mathrm{O}_{2}}
$$

at a uniform temperature, where $p_{\mathrm{O} 2}$ varied from 1 to $10^{-4}$ atm by mixing argon and air or oxygen. The exact partial pressure of oxygen in the mixture was monitored using an oxygen sensor based on yttria-stabilized zirconia.

All bulk properties discussed here, including resistance, transference number, and other related properties, are referred to as average properties of an MIEC in a concentration cell at a homogeneous temperature. The properties of bulk electrolyte usually vary with the concentrations of electroactive species. For a BCG electrolyte, for instance, its properties are strong functions of $p_{\mathrm{O}}$. However, it is the average properties which have practical significance because electrolyte materials often are exposed to a gradient in chemical potential for practical applications (such as in a solid oxide fuel cell or in a chemical sensor).

\section{Results and Discussion}

Effect of oxygen partial pressure--Shown in Fig. 1 are some typical impedance spectra of cell 1 at $722^{\circ} \mathrm{C}$. Both $R_{b}$ and $R_{\mathrm{T}}$, as determined from the impedance spectra, are a strong function of oxygen partial pressure, $p_{\mathrm{O}_{2}}$. Because of the electronic conduction in the material, the interfacial resistance cannot be determined simply from the impedance spectra alone. Instead, Eq. 6 to 8 must be used to calculate the resistances. Shown in Fig. 2 are the applied Nernst potentials and OCVs of the cell measured under the same conditions under which the impedance spectra were acquired.

Combination of the data shown in Fig. 1 and 2 allows accurate determination of $R_{\mathrm{i}}, R_{\mathrm{e}}$, and $R_{\mathrm{p}}$ using Eq. 6 to 8 . As clearly shown in Fig. 3, the resistance of the electrolyte-electrode interfaces $\left(R_{p}\right)$ increased rapidly as the $p_{\mathrm{O}_{2}}$ is reduced. This is expected because the interfacial polarizations due to both charge and mass transfer usually becomes more severe as $p_{\alpha_{2}}$ is reduced. Accordingly, the electrochemical processes occurring at or near the electrolyteelectrode interfaces can be investigated from the dependence of the interfacial resistance on $p_{\mathrm{O}_{2}}$ and on temperature.

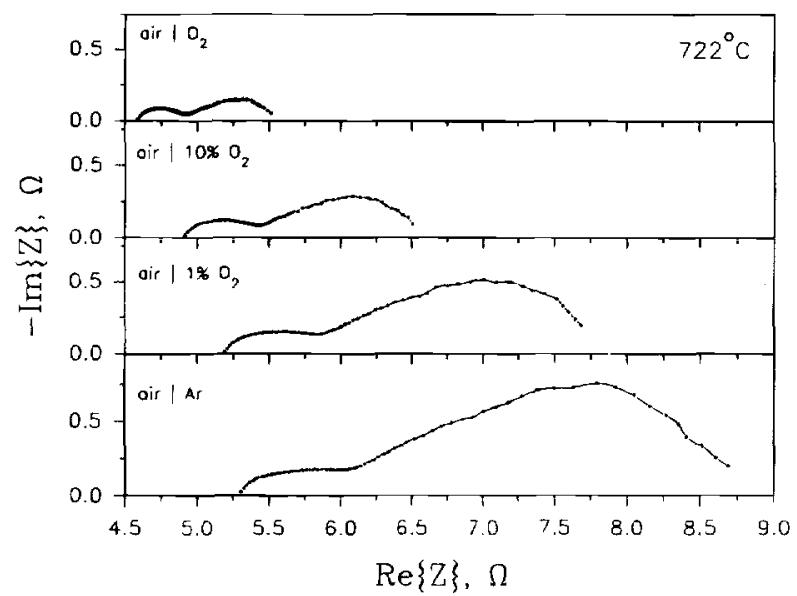

Fig. 1. Impedance spectra of cell 1 measured at $722^{\circ} \mathrm{C}$ (the electrolyte thickness was $1.2 \mathrm{~mm}$ and the electrode area was $0.91 \mathrm{~cm}^{2}$ ).

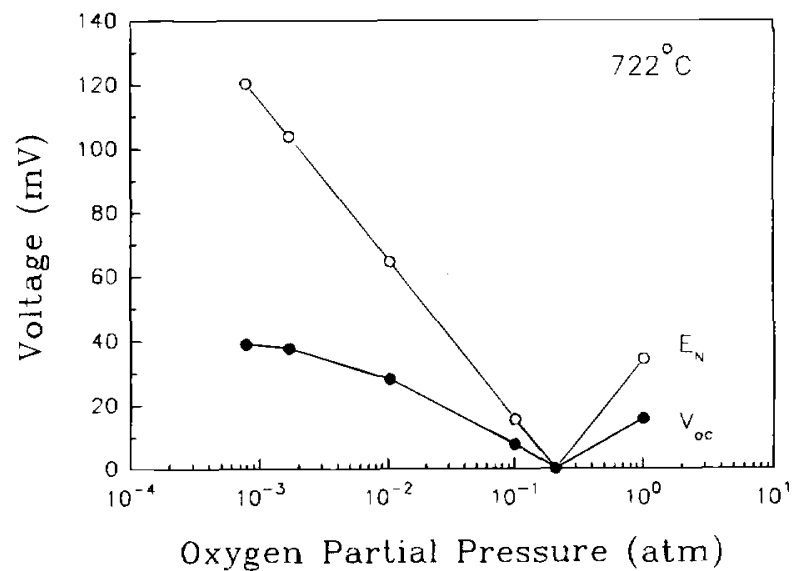

Fig. 2. Observed $\mathrm{OCV}$ and the applied Nernst potential across cell 1 under the conditions for the impedance measurements.

The ratios of $V_{\text {od }} / E_{\mathrm{N}}$ and the average ionic transference numbers calculated using Eq. 9 are summarized in Fig. 4. Clearly, the ratios of $V_{\mathrm{od}} / E_{\mathrm{N}}$ are different from the ionic transference numbers. At a constant temperature $\left(722^{\circ} \mathrm{C}\right)$, the deviation of ionic transference number from the ratio of $V_{0} J E_{N}$ becomes larger as the partial pressure of oxygen is reduced. It is also apparent from Fig. 3 and 4 that the larger the interfacial resistance, the larger is the relative error $\epsilon$ introduced by the use of Eq. 1.

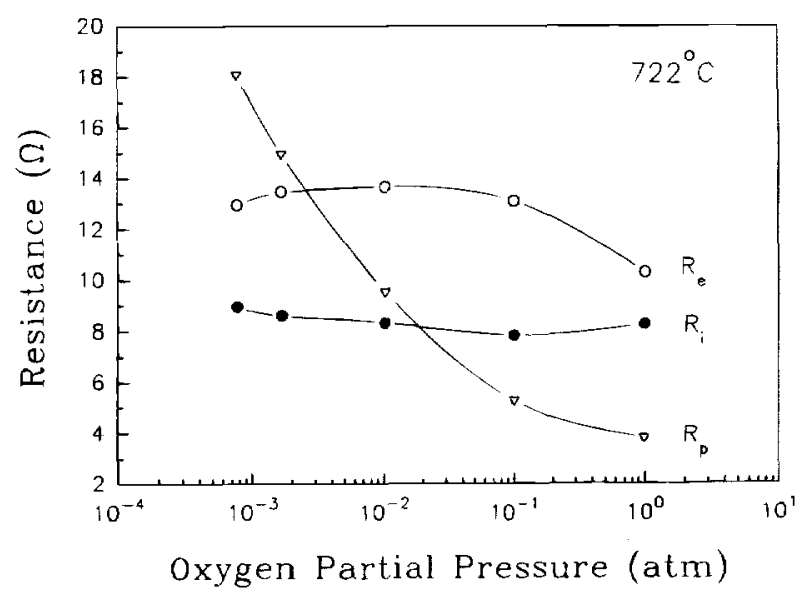

Fig. 3. Calculated average lonic and electronic resistances of the BCG electrolyte and the interfacial resistances of cell 1. 


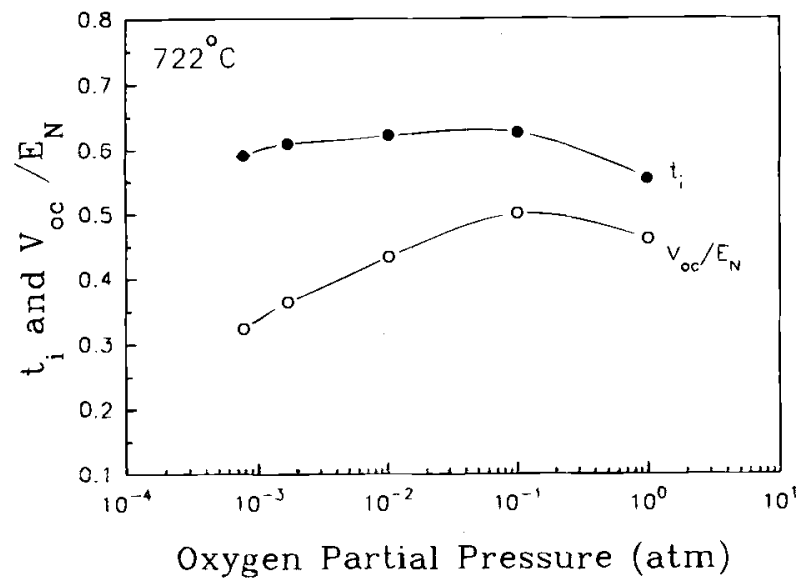

Fig. 4. The $V_{o} / E_{N}$ ratios and the calculated ionic transference numbers for the BCG electrolyte as determined in cell 1.

Effect of temperature.-Shown in Fig. 5 are some typical impedance spectra of cell 1 measured at different temperatures when one electrode of the cell was exposed to air while the other electrode was exposed to argon. Because the rate of oxygen permeation through the BCG electrolyte from the air side to the argon side depends on temperature, the actual partial pressure of oxygen in the chamber with argon varied with temperature, from $2.7 \times$ $10^{-4} \mathrm{~atm}$ at $620^{\circ} \mathrm{C}$ to $7.7 \times 10^{-4} \mathrm{~atm}$ at $722^{\circ} \mathrm{C}$, as indicated in Fig. 5 . Similar impedance data were acquired with the same cell at these temperatures but in an atmosphere containing different partial pressures of oxygen. The applied $E_{\mathrm{N}}$ and OCV measured under identical conditions for impedance measurements are shown in Fig. 6.

One simple yet powerful way to evaluate the validity of Eq. 1 is to examine the ratios of $R_{\mathrm{b}} / R_{\mathrm{T}}$ and $V_{\mathrm{od}} / E_{\mathrm{N}}$. Rearranging Eq. 4 and 5 yields

$$
\frac{R_{\mathrm{o}}}{R_{\mathrm{T}}}\left(\frac{R_{\mathrm{i}}+R_{\mathrm{p}}}{R_{\mathrm{i}}+R_{\mathrm{p}}+R_{\mathrm{e}}}\right)=\frac{R_{\mathrm{i}}}{R_{\mathrm{i}}+R_{\mathrm{e}}} \equiv t_{\mathrm{e}} \equiv 1-t_{\mathrm{i}}<\frac{R_{\mathrm{b}}}{R_{\mathrm{T}}}[11]
$$

The last inequality is a consequence of the fact that $R_{\mathrm{e}}>0$. If Eq. 1 is true, then

$$
1-\frac{V_{o c}}{E_{\mathrm{N}}}<\frac{R_{\mathrm{b}}}{R_{\mathrm{T}}}
$$

Analysis of the data in Fig. 5 and 6 (summarized in Table I) indicates, however, that the inequality 12 is invalid; the values of 1 $V_{\mathrm{oc}} E_{\mathrm{N}}$ are greater, not smaller, than the ratios of $R_{\mathrm{b}} / R_{\mathrm{T}}$ for all cases.

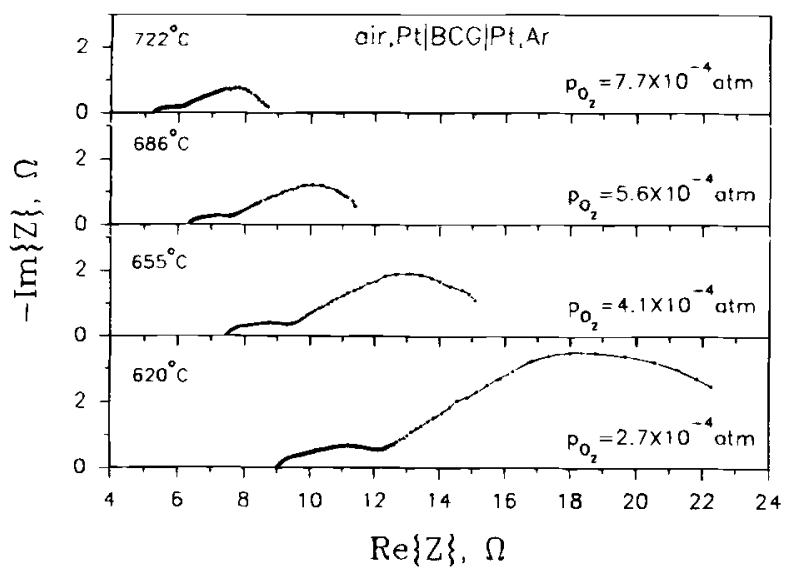

Fig. 5. Impedance spectra of cell 1 measured at different temperatures when one electrode was exposed to air while the other electrode was exposed to argon. The oxygen partial pressure adjacent to each spectrum represents the $p_{o_{2}}$ in the argon side of the cell at the temperature. The electrolyte thickness was $1.2 \mathrm{~mm}$ and the electrode area was $0.91 \mathrm{~cm}^{2}$.

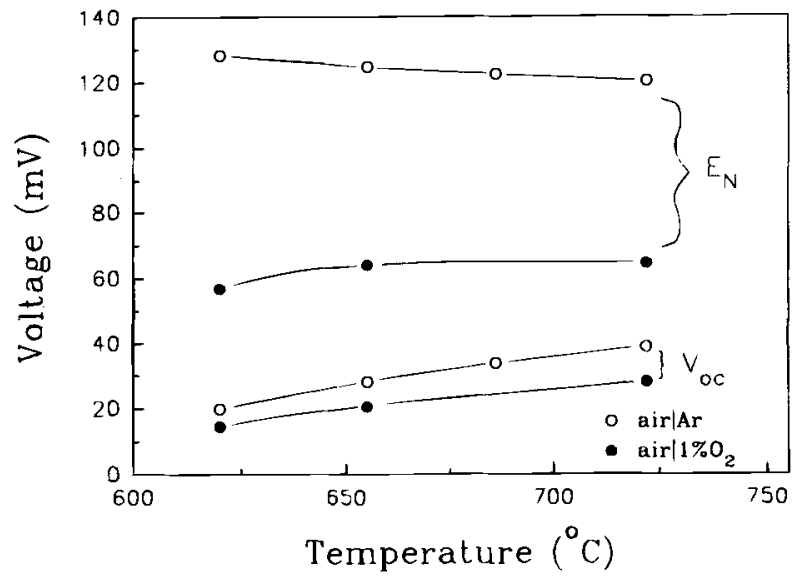

Fig. 6. Observed OCV and the applied Nernst potential across cell 1 under conditions similar to those described in Fig. 5.

This striking discrepancy is a direct consequence of the assumption that $t_{\mathrm{i}}=V_{\mathrm{o}} E_{\mathrm{N}}$, strongly suggesting that the ratio of $V_{\mathrm{o}} d E_{\mathrm{N}}$ deviates far from the average ionic transference number. Errors introduced by the use of Eq. 1 under these testing conditions are so large that the interfacial resistances defined earlier must take negative values for the equations involving $R_{\mathrm{p}}$ to hold. The corresponding relative error $\epsilon$ for each case is listed in Table 1 .

Shown in Fig. 7 are the calculated ionic and electronic resistances of the electrolyte and the interfacial polarization resistances of the cell at temperatures varying from 620 to $722^{\circ} \mathrm{C}$. Clearly, the activation energies for interfacial processes are much greater than those for the transport in the bulk electrolyte and, as a result, the interfacial resistance increases much faster than the bulk resistance of an electrolyte as temperature is reduced.

As shown in Fig. 8, the ionic transference numbers are far greater than the ratios of $V_{\mathrm{oc}} / E_{\mathrm{N}}$. For a given atmosphere, the deviation of the ionic transference number from the ratio of $V_{\mathrm{oc}} / E_{\mathrm{N}}$ increases as temperature is reduced.

Effect of cell geometry.-For a cell with electrolyte thickness $L$ and electrode area $S$, the geometry-independent properties of the electrolyte and the interfaces can be expressed as

$$
\begin{gathered}
\rho_{\mathrm{k}}=\frac{R_{\mathrm{k}} S}{L} \quad k=\mathrm{i} \text { or } \mathrm{e} \\
\rho_{\mathrm{p}}=R_{\mathrm{p}} S
\end{gathered}
$$

where $\rho_{i}$ and $\rho_{\theta}$ are the ionic and electronic resitivitiy $(\Omega \mathrm{m})$ of the bulk electrolyte and $\rho_{p}$ is the specific resistance $\left(\Omega \mathrm{m}^{2}\right)$ of the interfaces. In terms of $\rho_{i}, \rho_{e}$, and $\rho_{p}$, $\delta$ can be expressed as

$$
\delta=\left(\frac{\rho_{\mathrm{p}}}{\rho_{\mathrm{i}}+\rho_{\mathrm{e}}}\right)\left(\frac{1}{L}\right)
$$

Thus, theoretically speaking, $\delta$ or $\epsilon$ is independent of electrode area but becomes greater for a cell with a thinner electrolyte.

Table I. Ratios of $R_{\mathrm{b}} / R_{\mathrm{T}}$ and $V_{\mathrm{od}} / E_{\mathrm{N}}$ calculated from impedance spectra (Fig. 5) and OCV measurements (Fig. 6) of a cell with a configuration of air, PtIBCGIPt, argon.

\begin{tabular}{cccc}
$\begin{array}{c}\text { Temperature } \\
\left({ }^{\circ} \mathrm{C}\right)\end{array}$ & $R_{\mathrm{b}} / R_{\mathrm{T}}$ & $1-V_{\mathrm{oc}} / E_{\mathrm{N}}$ & $\epsilon=\delta /(1+\delta)$ \\
\hline 620 & 0.39 & 0.84 & 0.77 \\
655 & 0.48 & 0.77 & 0.64 \\
686 & 0.55 & 0.72 & 0.54 \\
722 & 0.60 & 0.67 & 0.45
\end{tabular}




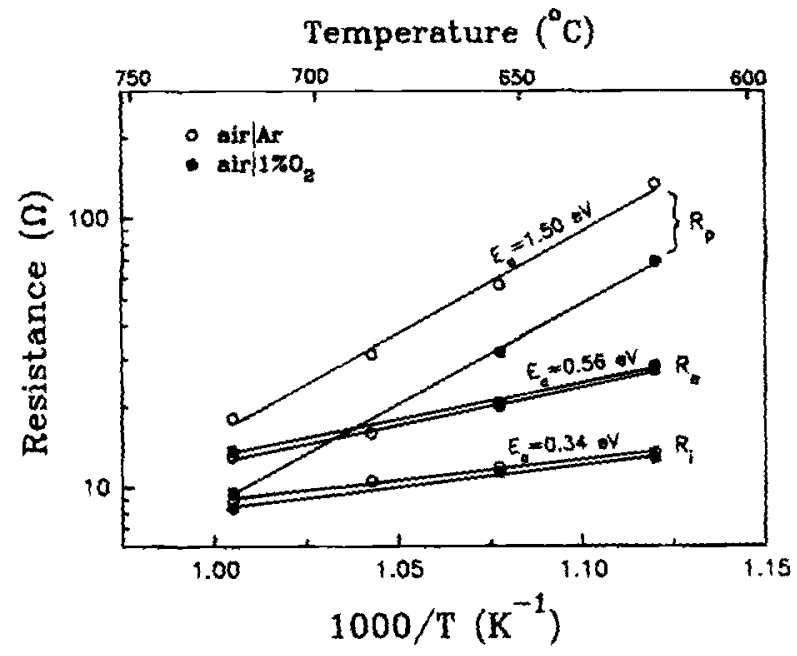

Fig. 7. Calculated ionic and electronic resistances of the BCG electrolyte and the interfacial resistances of cell 1 under conditions similar to those specified in Fig. 5.

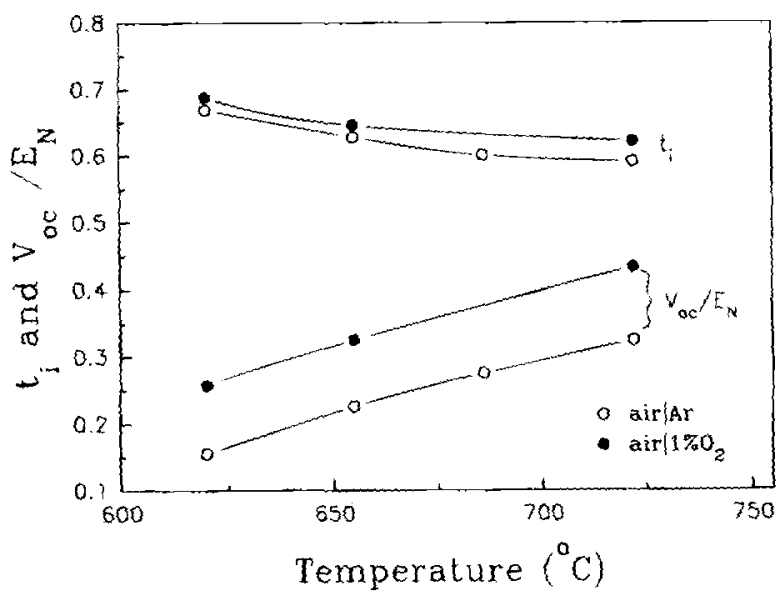

Fig. 8. The $V_{o} / E_{\mathrm{N}}$ ratios and the calculated ionic transference numbers for the BCG electrolyte under conditions similar to those specified in Fig. 5.

\section{Conclusion}

The dramatic effect of intertacial resistance on determination of the transport properties of an MEC has been clearly demonstrated. The observed ratio of $V_{o} / E_{N}$ for a mixed-conducting electrolyte can be very different from the average ionic transference number of the electrolyte; the ratio is a good approximation to the ionic transference number only when the interfacial processes are sufficiently fast or the interfacial resistance is much smaller than the bulk resistance of the electrolyte. Generally, the relative error intro- duced by ignoring the effect of interfacial resistance becomes more significant for cells based on thinner electrolytes, measured at lower temperatures, and exposed to an atmosphere containing lower activity of electroactive species.

\section{Acknowledgment}

This work was supported by EPRI under Contract No. RP 167619 and by NSF under Award No. DMR-9357520.

Manuscript received Feb. 1, 1996.

Georgia Institute of Technology assisted in meeting the publication costs of this article.

$E_{N} \quad$ Nernst potential, $V$

\section{LIST OF SYMBOLS}

F Faraday's constant, $96,487 \mathrm{C} \mathrm{eq}^{-1}$

$p_{\mathrm{O}_{2}} \quad$ partial pressure of oxygen

$R^{2} \quad$ universal gas constant, $8.314 \mathrm{~J} \mathrm{~mol}^{-1} \mathrm{~K}^{-1}$

$R_{0} \quad$ resistance of a bulk electrolyte, $\Omega$

$R_{e}$ resistance to the motion of electronic species in a bulk electrolyte, $\Omega$

$R_{3}$ resistance to the motion of ionic species in a buik electrolyte, $\Omega$

$R_{p} \quad$ intertacial polarization resistance of a cell, $\Omega$

$R_{\text {T }}$ total resistance of a cell, $\Omega$

$t_{0}$ electronic transference number, dimensionless

$t_{i}$ ionic transference number, dimensionless

$T$ absolute temperature, $K$

$V_{\infty}$ open-cell voltage, $V$

$\eta n$ anodic and cathodic overpotential, respectively, $V$

$\delta$ as defined by Eq. $3, \delta=R_{0}\left\langle\left(R_{i}+R_{e}\right)\right.$

e $\quad$ alative error as defined by $\mathrm{Eq} .10, \epsilon=\delta /(1+\delta)$

\section{REFERENCES}

1. H. Yahiro, Y. Baba, K. Eguchi, and H. Arai, This Journal, 135, 2077 (1988).

2. N. Bonanos, B. Ellis, K. S. Knight, and M. N. Mahmood, Solid State lonics, 35, 179 (1989).

3. H. Iwahara, ibid., 52, 99 (1992).

4. C. Wagner, Z. Phys. Chem., B21, 25 (1933).

5. M. Liu, H. Hu and W. Rauch, in Proceedings of the First international Symposium on Ceramic Membranes, $H . U$. Anderson, O. Yamamoto, A. C. Knandkar, M. Liu, and J.P. Dismukes, Editors, The Electrochemcial Society Proceedings Series, Pennington, NJ, In press (1996).

6. M. Liu, in Proceedings of the First International Symposium on lonic and Mixed Conducting Ceramics, T. A. Ramanarayanan and H. L. Tuller, Editors, FV 9\}-12, p. 191, The Electrochemica Society Proceedings Series, Pernington, NJ (1991).

7. M. Liu, Solid State lonics, To be submitted.

8. M. Liu and A. Joshi, in Proceedings of the First international Symposium on lonic and Mixed Conducting Ceramics, T. A. Ramanarayanan and $H$. L. Tulier, Editors, PV 91-12, p. 231. The Electrochemical Society Proceedings Series, Pennington, NJ (1991).

9. W. Rauch and $M$. Liu, in Role of Ceramics in Advanced Electrochemical Systems, P. N. Kumta, G. S. Rohrer, and U. Balachandran, Editors, Vol 65, The American Ceramic Society Transactions, Westerville, OH (1996). 